\title{
Comportamento Suicida e Religiosidade em estudantes de Psicologia
}

\author{
Maisa Carvalho Silva* \\ André Faro**
}

\begin{abstract}
Resumo
O presente estudo objetivou avaliar o comportamento suicida em estudantes de psicologia e analisar sua relação com a religiosidade. Participaram 129 estudantes de ambos os sexos com idades entre 17 e 53 anos, de diferentes períodos do curso de psicologia. Os instrumentos utilizados foram um questionário sociodemográfico, o Questionário de Comportamentos Suicidas Revisado (QCS-R) e o Índice de Religiosidade de Duke (DUREL). Os resultados mostraram que em discentes com idades entre 21 e 23 anos, 70,5\% afirmaram ter pensado ou tentado se matar, 24\% pensaram em se suicidar nos últimos 12 meses, 28,7\% comunicaram a intenção suicida a alguém e 11,6\% consideraram a possibilidade de se matar em um futuro próximo. Em relação à religiosidade, a dimensão intrínseca apresentou média de 8,28 (DP = 3,96). A hipótese da religiosidade como fator protetivo foi corroborada, visto que aparentou inibir ou reduzir o surgimento de comportamentos de risco ao suicídio.
\end{abstract}

Palavras-chave: Suicídio; Religiosidade; Universitários.

\section{Suicidal behavior and Religiosity in psychology students}

\begin{abstract}
The present study aimed to assess suicidal behavior in psychology students and to analyze its relationship with religiosity. Method: 129 students of both sexes Participated, aged between 17 and 53 years, from different periods of the psychology course. The instruments used were a sociodemographic questionnaire, the Revised Suicidal Behavior Questionnaire (QCS-R) and the Duke Religiosity Index (DUREL). The results showed that students aged between 21 and 23 years old, $70.5 \%$ said they had thought or tried to kill themselves, $24 \%$ thought about committing suicide in the last 12 months, $28.7 \%$ communicated the suicidal intention to someone and $11.6 \%$ considered the possibility of killing His or herself in the near future. Regarding religiosity, the intrinsic dimension showed an average of 8.28 (SD = 3.96). The hypothesis of religiosity as a protective factor was corroborated, since it appeared to inhibit or reduce the appearance of suicide risk behaviors.
\end{abstract}

Keywords: Suicide; Religiosity; College students.

\section{Introdução}

O Comportamento suicida é definido e expressado por uma série de elementos que podem desencadear o suicídio, tais como a ideação suicida, tentativas e o ato propriamente dito (Bertolote, Mello-Santos, \& Botega, 2010). O suicídio, fenômeno global responsável por aproximadamente 800 mil mortes anuais, é caracterizado como a $15^{\mathrm{a}}$ principal causa de morte e a segunda maior em indivíduos com idades entre 15-29 anos (World Health Organization - WHO, 2014), faixa etária correspondente ao ingresso de muitos jovens no ensino superior. No Brasil, são registradas cerca de 11 mil mortes anuais por suicídio e o fenômeno é tido como a quarta causa de

* Psicóloga pela Universidade Federal de Sergipe

** Professor Adjunto do PPG em Psicologia da Universidade Federal de Sergipe morte entre jovens com essa mesma faixa etária (Ministério da Saúde, 2019).

Em universitários, o autoextermínio é apontado como a segunda principal causa de morte (Lamis, Malone, \& Jahn, 2014) e vem sendo alvo de estudos ao redor do mundo em virtude do crescimento de óbitos observado nesse segmento populacional. Em um estudo desenvolvido por Santos, Marcon, Espinosa, Baptista e Paulo (2017) com uma amostra de universitários brasileiros, foi constatada uma prevalência de 9,9\% para ideação suicida em um período de 30 dias. Já o estudo realizado por O’Neill, McLafferty, Ennis, Lapsey, Bjourson, Armour e Murray (2018) com universitários da Irlanda do Norte identificou que aproximadamente $31 \%$ dos estudantes possuíam 
ideação suicida e quase 1 em cada 5 estudantes realizou um plano suicida nos 12 meses anteriores à pesquisa.

Tendo em vista que o suicídio possui um caráter multifatorial, não existe uma única ou maior causa para o comportamento suicida. Na literatura são listados alguns fatores de risco, tais como orientação sexual, ser do sexo masculino, presença de transtornos mentais, histórico de suicídio na família, realização de tentativas prévias, abuso sexual, problemas financeiros, características cognitivas e fácil acesso a meios letais (World Health Organization -WHO, 2014).

Os fatores de risco mais apontados ao comportamento suicida em universitários são dificuldades relacionadas à formação acadêmica, o desenvolvimento de transtornos mentais e o consumo de drogas (Pereira \& Cardoso, 2015). Um levantamento desenvolvido com graduandos de diversas instituições federais brasileiras detectou que dificuldades como a presença de sintomas ansiogênicos, adaptação a novas situações, dificuldade de aprendizado, timidez excessiva, medo/pânico, desânimo, sentimento de solidão, ideia de morte, ideação suicida, entre outros, podem ter interferido na vida acadêmica desses alunos (Associação Nacional dos Dirigentes das Instituições Federais de Ensino Superior - ANDIFES, 2016).

Os fatores protetivos, por sua vez, são aqueles que minimizam a probabilidade de ocorrer o suicídio. $\mathrm{O}$ suporte social, práticas religiosas, acesso reduzido a meios letais, estrutura familiar saudável, satisfação com a vida, autoestima e estilo cognitivo são alguns dos elementos que propiciam melhores formas de ajustamento frente às adversidades da vida (Centers for Disease Control and Prevention - CDC, 2017). Dentre os mencionados, a religiosidade é tida como um dos principais fatores de proteção e suporte contra uma série de condições, sejam elas clínicas ou não (Salas-Wright, Lombe, Nebbitt, Saltzman, \& Tirmazi, 2018).

A religiosidade é um construto multidimensional composto por crenças, práticas e rituais guiados por um ser superior e transcendental adotados pelo indivíduo para direcionar seus comportamentos, relações e modo de viver (Koenig, 2012). Difere da espiritualidade em termos conceituais e teóricos, sendo a primeira compreendida como um fenômeno institucional enquanto a segunda se remete a uma relação pessoal e subjetiva com o transcendente, podendo incluir alguma religião ou ir além dela (Jahn \& Dell'Aglio, 2017). Neste estudo, para fins de esclarecimento, utilizou-se o construto da religiosidade. Em saúde, sabe-se que a religiosidade funciona como um importante fator protetivo e de enfrentamento, o que contribui para aspectos favoráveis à saúde mental e física (Oliveira \& Junges, 2012). Um estudo desenvolvido por Fleury, Gomes, Rocha, Formiga, Marques e Bernardes (2018) com 588 universitários brasileiros encontrou que a religiosidade exerce uma influência positiva e significativa com a satisfação com a vida e também com a adoção de estratégias de enfrentamento. Outra pesquisa conduzida com 242 estudantes do curso de farmácia de uma universidade da Arábia Saudita evidenciou que a religiosidade pode aumentar a satisfação com a vida e aliviar o estresse acadêmico (Aftab, Naqvi, Al-karasneh, \& Ghori, 2018).

Quanto ao comportamento suicida, evidências empíricas indicam a religiosidade como um fator protetivo relevante (Dransart, 2018; Santos, Ulisses, Costa, Farias, \& Moura, 2016). Do contrário, estudo realizado por Lawrence, Brent, Mann, Grunebaum, Galfalvy e Oquendo (2016) com pacientes depressivos refere a religiosidade como um fator de risco para o suicídio, sugerindo uma relação complexa entre esses dois construtos. Outro estudo conduzido por Kralovec, Fartacek e Fartacek (2012) com lésbicas, gays e bissexuais austríacos encontrou que a religiosidade pode servir tanto como um fator protetivo quanto de risco ao fenômeno nessa população. Todavia, pouco se sabe sobre a interrelação negativa das práticas religiosas com o suicídio, sendo necessários novos estudos que reforcem ou desmistifiquem essa ideia.

Diante do panorama exposto, o objetivo geral deste estudo foi aferir os níveis de comportamento suicida entre os estudantes de Psicologia e, de forma específica, relacioná-los com a avaliação da religiosidade por entendê-la como um importante fator protetivo contra o suicídio.

\section{Método}

\section{Participantes}

A amostra, do tipo não-probabilística e de conveniência, foi constituída de 129 discentes, de ambos os sexos, com idades entre 17 e 53 anos, de diferentes períodos do curso de Psicologia de uma universidade pública localizada no Estado de Sergipe (Brasil). Participaram os alunos matriculados no curso, sendo este o único critério de inclusão estabelecido. A distribuição por período do curso ficou em $27,9 \%(n=36)$ para o $2^{\circ}$ período, $24 \%(n$ = 31) para o $4^{\circ}, 17,8 \%(n=23)$ para o $6^{\circ}, 14 \%(n=18)$ para o $8^{\circ}, 1,6 \%(n=2)$ para o $9^{\circ}, 10,9 \%(n=14)$ para o $10^{\circ}$ e $3,9 \%(n=5)$ para alunos sem período definido.

\section{Instrumentos}

Para a caracterização dos participantes, foi utilizado um breve questionário sociodemográfico constituído pelas variáveis sexo, idade e período do curso. 
A avaliação do comportamento suicida foi feita através do Questionário de Comportamentos Suicidas Revisado (Suicide Behavior Questionnaire Revised QCS-R), validado por Osman, Bagge, Gutierezz, Konick, Kopper e Barrios (2001). Composto por 4 itens em formato Likert com até seis níveis de resposta, demanda que o indivíduo responda sobre a existência de ideação suicida ou tentativa de suicídio em algum momento da vida, frequência de ideações no último ano, se a intenção já foi manifestada a alguém e se existe a possibilidade real do suicídio vir a ocorrer. Em virtude da proporção das respostas bastante desigual nos itens da escala Likert originalmente proposta, na análise de dados desta pesquisa se optou por dicotomizar os escores de resposta da escala em todos seus itens. Neste estudo a consistência interna alcançada foi considerada adequada $(\alpha=0,80)$.

Para a avaliação da religiosidade empregou-se o Índice de Religiosidade de Duke (DUREL), desenvolvido por Koenig, Meador e Parkerson (1997) e validado no Brasil por Taunay, Gondim, Macêdo, Moreira-Almeida, Gurgel, Andrade e Carvalho (2012). O instrumento possui cinco itens que captam três dimensões centrais da religiosidade relacionadas a desfechos em saúde, a saber: religiosidade organizacional $(\mathrm{RO})$, religiosidade não-organizacional (RNO) e religiosidade intrínseca (RI). Em virtude dos escores terem sido originalmente propostos em escala decrescente, optou-se pela recodificação de modo crescente dos itens de resposta a fim de facilitar a análise e interpretação dos resultados. Neste estudo a escala obteve boa consistência interna $(\alpha=0,93)$.

\section{Procedimentos e Aspectos éticos}

A pesquisa foi aprovada pelo Comitê de Ética (CAAE: 00618918.7.0000.5546) e se desenvolveu conforme os princípios éticos postulados pela resolução 510/2016 do Conselho Nacional de Saúde/Ministério da Saúde. Após aprovação, os dados foram coletados nas salas de aula dos indivíduos pretendidos sob a concessão dos docentes responsáveis e em outras imediações da instituição mencionada. Explicitado o tema a ser estudado, foram distribuídos e explicados os Termos de Consentimento Livre e Esclarecido (TCLE).

\section{Análise de Dados}

Os dados foram tratados e analisados pelo programa SPSS (Statistical Package for Social Sciences, versão 23). Foram computadas análises descritivas (média e desvio-padrão) e de frequência (absoluta e percentual) para todos os instrumentos. As estatísticas inferenciais foram obtidas através da execução do teste t de Student relacionando fatores do DUREL com a variável sexo, bem como os itens do QCS-R com a idade. O teste do Qui-Quadrado $\left(X^{2}\right)$ foi aplicado para analisar a relação entre o QCS-R e a variável sexo. A correlação de Tau B de Kendall foi aplicado para relacionar os fatores do DUREL com a idade. O nível de significância adotado foi o de $\mathrm{P}$-valor menor que 0,05 .

\section{Resultados}

Nesta amostra, $72,1 \%(n=93)$ de indivíduos eram do sexo feminino, com idade média de 23,3 anos ( $D P=$ 6,99). Para simplificar a descrição da idade dos participantes, optou-se por estratificar a variável apenas para essa finalidade. Sendo assim, por faixas etárias, 11,6\% ( $n$ $=15)$ dos participantes tinham idade entre 17 a 18 anos; $13,2 \%(n=17), 17,8 \%(n=17)$ e $17,8 \%(n=17) 19,20$ e 21 anos, respectivamente; $16,3 \%(n=21)$ entre $22-23$ anos e $23,3 \%(n=30)$ entre $24-53$ anos. Na Tabela 1 constam os resultados descritivos originais e agrupados do QCS-R. Dentre os principais dados relacionados aos itens não agrupados do QCS-R, considerando as maiores e menores frequências, verificou-se que para o item QCS-R (1a), 43,4\% $(n=56)$ dos discentes já tiveram um breve pensamento e 6,2\% [3,9\%(n=5) + 2,3\%(n= 3)] já tentaram o suicídio. No QCS-R (2), 58,1\% $(n=75)$ referiram que nunca pensaram e 3,9\% $(n=5)$ pensam frequentemente em se matar. Para o QCS-R (3) 71,3\% $(n=92)$ dos alunos nunca manifestaram para alguém o desejo de se suicidar e apenas 4,7\% $(n=6)$ disseram uma vez e desejavam que isso acontecesse. Por fim, no QCS-R (4) 35,7\% (n= 46) consideram bastante improvável e $1,6 \%(n=2)$ bastante provável cometer suicídio.

Quanto aos principais resultados dos itens agrupados (dicotomizados) do QCS-R, 70,5\% $(n=91)$ dos indivíduos já possuíram ideações suicidas e/ou tentaram se suicidar [QCS-R (1)], 76\% $(n=98)$ não pensaram em se matar no último ano [QCS-R (2)], 71,3\% $(n=92)$ nunca manifestaram para alguém a intenção de que iria ou poderia vir a se suicidar [QCS-R (3)], 88,4\% $(n=$ 114) consideram improvável de que poderiam cometer suicídio algum dia [QCS-R (4)], todavia, 11,6\% $(n=15)$ destes acreditam que seja provável tentarem suicídio em um futuro próximo [QCS-R (4)].

No que se refere à análise descritiva dos itens em escala de resposta nominal do DUREL, para o DUR (1) $(M=2,67 ; D P=1,61)$, os principais dados foram que $29,5 \%(n=38)$ dos participantes nunca frequentam instituições religiosas e apenas $7 \%(n=9)$ uma vez na 
semana. No DUR (2) $(M=2,75 ; D P=1,79)$, destaca-se que $41,1 \%(n=53)$ dos indivíduos raramente ou nunca se dedicam a essas atividades e 4,7\% $(n=6)$ uma vez por semana. No DUR (3) $(M=3,22 ; D P=1,50), 26,4 \%(n$ $=34)$ consideram ser totalmente verdade que sentem a presença de Deus ou do Espírito Santo e 9,3\% $(n=12)$ acreditaram que em geral não é verdade. No DUR (4) ( $M$ $=2,63 ; D P=1,36)$, para $30,2 \%(n=39)$ não é verdade e $9,3 \%(n=12)$ dos indivíduos tomam a afirmação como totalmente verdade. Já no DUR (5) $(M=2,42 ; D P=$ $1,45) 40,3 \%(n=52)$ não se esforçam e para $10,9 \%(n=$ 14) é totalmente verdade.

Os resultados categorizados do DUREL mostraram que a dimensão de religiosidade organizacional (F1) obteve pontuação média de 2,67 ( $D P=1,61)$, evidenciando que os indivíduos exercem a religiosidade em instituições uma vez por ano ou menos. Quanto à religiosidade não- -organizacional (F2), de média 2,75 ( $D P=1,79)$, viu-se que a amostra estudada exerce atividades religiosas individuais poucas vezes por mês. Por fim, a religiosidade intrínseca (F3), relacionada à percepção e manifestação individual da religiosidade na vida do indivíduo, obteve média de 8,28 ( $D P=3,96)$, o que representa elevada religiosidade nesse fator.

Na comparação entre os itens agrupados do QCS-R e o sexo não foi observada significância estatística para nenhum dos quatro itens $(p>0,05)$. Quanto à idade, três itens do QCS-R exibiram diferenças estatisticamente significativas. No item QCS-R (1), aqueles que nunca pensaram em tirar a própria vida eram mais velhos (Nunca: $M=25,5, D P=8,50$ ) que aqueles que afirmaram terem tido ideação ou tentativa $(M=22,3$; $D P=3,07)[t(127)=2,092 ; p=0,012]$. No QCS-R (2), aqueles que não pensaram em tirar a vida no último ano

Tabela 1 - Dados descritivos e agrupados do Questionário de Comportamentos Suicidas Revisado (QCS-R)

\begin{tabular}{|c|c|c|}
\hline QCS-R & Níveis de resposta & $\mathrm{F} \%(\mathrm{n}=129)$ \\
\hline \multirow{5}{*}{$\begin{array}{l}\text { QCS-R (1) } \\
\text { Já pensou alguma vez em se matar } \\
\text { ou tentou se matar? }\end{array}$} & Nunca & $29,5(38)$ \\
\hline & Tive apenas um breve pensamento passageiro & $43,4(36)$ \\
\hline & Tive um plano para me matar, pelo menos uma vez, mas não o tentei fazer & $20,9(27)$ \\
\hline & Tentei matar-me, mas não queria morrer & $3,9(5)$ \\
\hline & Tentei matar-me e esperava mesmo morrer & $2,3(3)$ \\
\hline \multirow{2}{*}{ QCS-R(1)* } & Nunca & $29,5(38)$ \\
\hline & Ideação e tentativa & $70,5(91)$ \\
\hline \multirow{5}{*}{$\begin{array}{l}\text { QCS-R }(2) \\
\text { Com que frequência pensou em se } \\
\text { matar no último ano? }\end{array}$} & Nunca & $58,1(75)$ \\
\hline & Raramente (1 vez) & $17,8(23)$ \\
\hline & Algumas vezes (2 vezes) & $11,6(15)$ \\
\hline & Frequentemente ( 3 ou 4 vezes) & $3,9(5)$ \\
\hline & Muito frequentemente (5 ou mais vezes) & $8,5(11)$ \\
\hline \multirow{2}{*}{ QCS-R $(2)^{*}$} & Nunca (Nunca e Raramente) & $76(98)$ \\
\hline & Algumas vezes (Algumas vezes, Frequentemente e Muito frequentemente) & $24(31)$ \\
\hline \multirow{5}{*}{$\begin{array}{l}\text { QCS-R (3) } \\
\text { Já disse a alguém que iria se } \\
\text { suicidar ou que poderia vir a se } \\
\text { suicidar? }\end{array}$} & Não & $71,3(92)$ \\
\hline & Sim, uma vez, mas não queria realmente morrer & $12,4(16)$ \\
\hline & Sim, uma vez, e queria realmente morrer & $4,7(6)$ \\
\hline & Sim, mais do que uma vez, mas não queria fazê-lo & $5,4(7)$ \\
\hline & Sim, mais do que uma vez, e queria realmente fazê-lo & $6,2(8)$ \\
\hline \multirow{2}{*}{ QCS-R $(3)^{*}$} & Não & $71,3(92)$ \\
\hline & Sim (Todos os níveis de resposta positivos) & $28,7(37)$ \\
\hline \multirow{5}{*}{$\begin{array}{l}\text { QCS-R (4) } \\
\text { Qual a probabilidade de poder vir a } \\
\text { se suicidar um dia? }\end{array}$} & Nunca & $15,5(20)$ \\
\hline & Nenhuma possibilidade & $15,5(20)$ \\
\hline & Bastante improvável & $35,7(46)$ \\
\hline & $\begin{array}{l}\text { Improvável } \\
\text { Provável }\end{array}$ & $\begin{array}{ll}21,7 & (28) \\
10,1(13) \\
\end{array}$ \\
\hline & Bastante provável & $1,6(2)$ \\
\hline \multirow{2}{*}{ QCS-R (4)* } & Improvável (Nunca, nenhuma possibilidade, bastante improvável e improvável) & $88,4(114)$ \\
\hline & Provável (Provável e bastante provável) & $11,6(15)$ \\
\hline
\end{tabular}


também eram mais velhos $(M=23,8, D P=7,69)$ que aqueles que pensaram algumas vezes $(M=21,7, D P=$ $3,78)[t(127)=1,999 ; p=0,008]$. O QCS-R (3) não exibiu diferenças estatisticamente significativas em relação à idade $(p>0,05)$. Por fim, no QCS-R (4), foram mais velhos aqueles que disseram ser improvável vir a tirar a própria vida um dia $(M=23,5, D P=7,35)$, isso em comparação àqueles que afirmaram ser provável $(M=$ $21,4, D P=2,74)[t(127)=2,110 ; p=0,035]$. Noutras palavras, os participantes que possuem idades entre 21 e 23 anos tenderam a afirmar ideação ou tentativa de suicídio, terem pensado em se matar por algumas vezes no último ano, manifestado a alguém o desejo de se suicidar e também acreditam que sejam mais prováveis a tentar o suicídio algum dia.

$\mathrm{Na}$ relação entre os fatores do DUREL e os itens agrupados do QCS-R, foram observadas diferenças estatisticamente significativas com diferentes itens (Tabela 2). Viu-se que na relação com o QCS-R (1), apenas o F1 do DUREL apresentou diferença significativa $[t(127)=$ $3,251 ; p=0,011]$, em que "nunca" $(M=3,42 ; D P=1,78)$ obteve maior pontuação média que "ideação e tentativa" $(M=2,36 ; D P=1,43)$. Quanto aos F2 e F3, não houve significância estatística $(\phi>0,05)$. Os três fatores do DUREL e o QCS-R (2) relacionaram-se de modo estatisticamente significativo [F1: $t(127)=3,032$ e $p=0,011$; F2: $t(127)=3,731$ e $p=0,001 ; \mathrm{F} 3: t(127)=4,475$ e $p$ $=0,001]$, com maiores médias para "nunca" em todos os fatores $(M=2,86, D P=1,69 ; M=3,02, D P=1,85$ e $M$ $=8,96, D P=4,07$, respectivamente) em comparação às médias alcançadas por "algumas vezes" $(M=2,06, D P$ $=1,12 ; M=1,90, D P=1,30$ e $M=6,12, D P=2,69$, respectivamente). Para o QCS-R (3) foi notada significância apenas com o F2 do DUREL $[t(127)=2,322 ; p$ $=0,008]$, com maior média para "não" $(M=2,96, D P$ $=1,84)$ do que "sim" $(M=2,21, D P=1,58)$, denotando relação entre pouca ou nenhuma comunicação sobre a intenção suicida com manifestações de religiosidade não-organizacionais. Por fim, comparando-se o QCS-R (4) com os fatores do DUREL, todas as diferenças entre médias se apresentaram estatisticamente significativas [F1: $t(127)=4,620$ е $p=0,013 ; \mathrm{F} 2: t(127)=2,520$ е $p$ $=0,014$; F3: $t(127)=3,912$ e $p=0,010]$, com maiores médias para "improvável" em todas as relações $(M=$ $2,81, D P=1,63 ; M=2,87, D P=1,80$ e $M=8,62, D P$ $=4,01$, respectivamente) em comparação com os valores obtidos por "provável" $(M=1,60, D P=0,82 ; M=1,80$, $D P=1,52$ e $M=5,73, D P=2,46$, respectivamente).
Tabela 2 - Dados acerca da relação entre o Questionário de Comportamento Suicidas Revisado e o Índice de Religiosidade de DUKE

\begin{tabular}{|c|c|c|c|c|}
\hline \multirow{2}{*}{ F1 } & \multirow{2}{*}{ QCS-R } & \multicolumn{3}{|c|}{ DUREL } \\
\hline & & $\mathrm{F} 2$ & F3 & \\
\hline \multirow{2}{*}{ QCS-R (1) } & Nunca & $3,42(1,78)^{a}$ & $3,28(1,90)$ & $9,65(4,25)$ \\
\hline & Ideação e tentativa & $2,36(1,46)$ & $2,52(1,71)$ & $7,71(3,71)$ \\
\hline \multirow{2}{*}{ QCS-R (2) } & Nunca & $2,86(1,69)^{\mathrm{b}}$ & $3,02(1,85)^{\mathrm{b}}$ & $8,96(4,07)^{b}$ \\
\hline & Algumas vezes & $2,06(1,12)$ & $1,90(1,30)$ & $6,12(2,96)$ \\
\hline \multirow{2}{*}{ QCS-R (3) } & $\operatorname{Sim}$ & $2,82(1,62)$ & $2,96(1,84)^{c}$ & $8,77(3,79)$ \\
\hline & Não & $2,29(1,52)$ & $2,21(1,58)$ & $7,08(4,18)$ \\
\hline \multirow{2}{*}{ QCS-R (4) } & Improvável & $2,81(1,63)^{\mathrm{d}}$ & $2,87(1,80)^{\mathrm{d}}$ & $8,62(4,01)^{d}$ \\
\hline & Provável & $1,60(0,82)$ & $1,80(1,52)$ & $5,73(2,46)$ \\
\hline
\end{tabular}

Notas: ${ }^{\mathrm{a}} p=0,011 ;{ }^{\mathrm{b}} \mathrm{F} 1: p=0,011 ; \mathrm{F} 2: p=0,001$ e F3: $p=0,001 ;{ }^{\mathrm{c}} \mathrm{F} 2: p=$ 0,$008 ;{ }^{\mathrm{d}} \mathrm{F} 1: p=0,013 ; \mathrm{F} 2: p=0,014$ e F3: $p=0,010$

\section{Discussão}

O presente estudo teve predominância de mulheres, estudantes de períodos iniciais e, de maneira geral, jovens adultas. Tais achados são condizentes com dados atuais fornecidos pelo Conselho Federal de Psicologia (2019), pois, dos 333.596 profissionais da área, a maior parte é composta por mulheres, sendo esta uma tendência vista nos cursos de graduação (Macedo, Alves, Bezerra, \& Silva, 2018).

A partir da análise do QCS-R (1) não agrupado, viu-se que esta amostra obteve frequências consideradas altas no que se refere a pensamentos breves sobre se suicidar e, sobretudo, para tentativas. Por exemplo, ao realizar o cálculo da razão do número de participantes pelo total de pessoas que afirmaram já ter tentado o suicídio, obteve-se que para cada 16 alunos desta amostra, um deles já tentou o suicídio. Ainda que não seja uma comparação plenamente crível em termos epidemiológicos, mas que pode servir como parâmetro de reflexão nesta investigação. No ano de 2016 a taxa de mortes por suicídio chegou a 5,5 casos por 100 mil habitantes na população brasileira em geral (Ministério da Saúde, 2019). Ou seja, convertendo para uma razão simplificada, seria aproximadamente 1 caso $(1,16)$ para cada 20.000 habitantes. Portanto, comparando-se as taxas, o grau de risco para o suicídio pode ser considerado alto nesta amostra.

No mesmo item agrupado, foi possível evidenciar ainda mais a prevalência do comportamento suicida nesta amostra. Ao agrupar os níveis de resposta, a frequência percentual de ideação e tentativa de suicídio totalizou pouco mais de $70 \%$. Ao calcular a razão do total de 
participantes pelo número de pessoas que afirmaram possuir ideação ou ter tentado suicídio, o resultado aponta que, no geral, quase todos os participantes já pensaram em se matar ou tentaram suicídio (1 para 1,41 pessoas). Pesquisas recentes que objetivaram identificar a presença de ideação suicida em universitários encontraram dados semelhantes no que concerne as altas taxas de ideação, ressaltando ser este um problema atual, de caráter multifatorial e crescente no âmbito acadêmico (O’Neill et al., 2018; Santos, Marcon, Espinosa, Baptista, \& Paulo, 2017).

O item QCS-R (2) agrupado mediu a frequência das ideações nos últimos 12 meses e obteve uma frequência considerada alta (24\%), apontando que, aproximadamente, para cada 4 alunos 1 deles pensou em se matar algumas vezes no último ano; sugerindo, então, o suicídio como um tema não tão raro na vida desses alunos. Fazendo-se um paralelo, um estudo realizado em 2014 com alunos de diversas instituições federais brasileiras identificou que 4,13\% ( $n=38.838$ de 940 mil) dos universitários apresentaram ideações suicidas no último ano (ANDIFES, 2016).

Entende-se que tais achados servem como sinais de alerta para a necessidade de intervenções preventivas específicas com o público que apresenta maior vulnerabilidade, como é o caso da ideação suicida. Além das inespecíficas, promoção de saúde, com o restante da comunidade estudantil para conectar o estudante a serviços de suporte e tratamento em caso de eventual necessidade. Vale enfatizar que as intervenções em atenção primária são relevantes em termos de saúde pública, visto que a trajetória estabelecida entre a ideação suicida e o suicídio consumado pode permitir tempo hábil para intervenções (Braga \& Dell'Aglio, 2013).

A variável relacionada à comunicação da intenção suicida [QCS-R (3) agrupado] apresentou um dado interessante, visto ser uma especificidade pouco analisada na literatura. Nesta amostra, 28,6\% (aproximadamente 1 para cada 3 participantes) afirmaram já ter comunicado a alguém que iria ou poderia vir a se suicidar, evidenciando que a amostra estudada aparenta ser mais aberta a falar sobre o problema. A proporção encontrada foi maior que em um estudo realizado com jovens suicidas de uma comunidade vietnamita, em que 7 dos 19 participantes explicitaram verbalmente a amigos e conhecidos o desejo de se suicidar (Wasserman, Thanh, Minh, Goldstein, Nordenskiöld, \& Wasserman, 2008).

Embora a comunicação por si só não tenha o poder de prevenir a maioria dos suicídios, essa sinalização se apresenta como um aspecto positivo, pois aqueles indivíduos falam sobre seu sofrimento psíquico para outrem passam a ser mais passíveis de receberem suporte social e profissional (McCabe, Garside, Backhouse, \& Xanthopoulou, 2016). Outro ganho a partir do dado relativo à comunicação do ato se refere à desmistificação da crença comum de que o suicídio acontece sem avisos prévios (WHO, 2014).

Quanto à probabilidade de tentar suicídio em um futuro próximo [QCS-R (4) agrupado], encontrou-se que para cada 8 alunos, 1 deles considera provável tentar suicídio. Um estudo similar realizado com 1704 universitários identificou uma ocorrência de 3,4\% para este mesmo item do QCS-R (Becker, Holdaway, \& Luebbe, 2018). Convertendo os valores encontrados neste estudo para uma razão simplificada, estima-se que de cada 46 graduandos, 1 deles pensa em se matar algum dia. Frente a esse tipo de problemática, a Associação Brasileira de Psiquiatria [ABP] (2014) sugere ações preventivas como a problematização e educação permanente sobre o tema, criação de grupos de apoio e uso estratégico da mídia como ações preventivas. Para fins de ilustração da importância de ações desse tipo, um programa de prevenção utilizado em uma cidade do interior do Rio Grande do Sul com altos coeficientes de mortalidade para o suicídio mostrou-se eficaz ao reduzir a taxa de 5,0 pra 3,6 óbitos/ ano ao longo de 13 anos (Conte, Meneghel, Trindade, Ceccon, Hesler, Cruz \& Jesus, 2012).

No que se refere ao perfil de religiosidade, os participantes deste estudo demonstraram frequentar instituições religiosas com pouca periodicidade e realizar poucas atividades religiosas individuais. Em contraparti$\mathrm{da}$, internalizam mais a religiosidade, relacionando-a com a presença de entes espirituais. Uma pesquisa realizada com graduandos de enfermagem que objetivou coletar opiniões acerca da relação entre saúde, religiosidade e espiritualidade também encontrou índices análogos para a religiosidade intrínseca (Espinha, Camargo, Silva, Pavelqueires, \& Lucchetti, 2013). Tais dados revelam que a percepção individual sobre a religiosidade e a importância desta na vida do indivíduo apresenta, ainda que minimamente, maior relevância que frequentar uma instituição religiosa ou realizar atividades religiosas descentralizadas.

Quanto à relação com a idade, os mais jovens apresentaram maiores associações com o comportamento suicida. Isso se relaciona, de certo modo, ainda que com ressalvas, aos estudantes dos períodos iniciais da graduação. É importante salientar que o suicídio é a segunda causa de óbitos em indivíduos com idade entre 15-29 anos, período que eventualmente coincide com o 
ingresso de muitos jovens na universidade (CDC, 2017). O jovem recém-chegado à universidade, por vezes, dispõe de poucos recursos para lidar com as mudanças de vida proporcionadas pelo ingresso na academia e pode ver no suicídio uma alternativa para cessar as dificuldades. Tal hipótese foi reforçada por uma pesquisa realizada com universitários chineses em que foram encontradas associações entre o comportamento suicida, as experiências adversas na infância e os eventos de vida estressores relacionados à academia (You, Chen, Yang, Zhou, \& Qin, 2014).

Sobre a relação entre a religiosidade e o comportamento suicida nessa amostra, exercer a religiosidade em algum nível, seja ele organizacional, não-organizacional ou intrínseco, atuou como um fator protetivo ao suicídio, tendo isso sido evidenciado nas relações com todos os itens do QCS-R. Possuir ideação suicida ou já ter tentado se matar se associou com a religiosidade organizacional, demonstrando que indivíduos que frequentem assiduamente alguma instituição religiosa podem possuir menos chances de apresentar comportamento suicida. Por outro lado, não pensar em se suicidar nos últimos 12 meses foi associado a todas as dimensões de religiosidade. Além disso, comunicar a pretensão de tentar suicídio se relacionou positivamente apenas com a dimensão não-organizacional, evidenciando que ambientes não-institucionalizados parecem ser mais abertos e acolhedores para esse tipo de questão. Quanto à probabilidade futura de tentar o suicídio, esta correlacionou-se com todos os níveis de religiosidade. Noutras palavras, nesta amostra, professar a religiosidade em qualquer um dos níveis mencionados se configurou como um fator protetivo quanto à possibilidade de tentar suicídio algum dia.

Há indícios de que possuir uma prática religiosa se constitui como um importante fator protetivo em saúde e costuma atuar como uma estratégia de enfrentamento para uma série de condições clínicas, além de estar associada a bons índices de qualidade de vida, bem-estar e outros benefícios (Fleury et al., 2018). Embora os resultados deste trabalho tenham associado a religiosidade com a menor prevalência de comportamentos suicidas, vale dizer que esta hipótese ainda é inconsistente na literatura científica (Jongkind, Van den Brink, Schaap-Jonker, Van der Velde, \& Braam, 2018). Inclusive, um estudo aponta que a religiosidade não é eficaz como fator protetivo à ideação suicida, apenas frente às tentativas e, possivelmente, ao suicídio consumado (Lawrence, Oquendo, \& Stanley, 2016).

\section{Conclusão}

Em síntese, os principais achados deste estudo apontaram para índices preocupantes de ideação, tentativas e intenções futuras de suicídio entre os alunos mais jovens do curso de Psicologia. Entretanto, encontrou-se achados interessantes relativos à comunicação do ato, caso se considere a possibilidade de intervenções. A hipótese de que a religiosidade se expressaria como um fator de proteção contra o comportamento suicida foi corroborada, constatando-se que a prática religiosa aparentou inibir ou reduzir o surgimento de comportamentos de risco ao suicídio.

Quanto às limitações metodológicas, cita-se o número reduzido de participantes. Ainda que tenha alcançado cerca de $50 \%$ da população-alvo, acaba por limitar a generalização dos resultados, especialmente por ter sido aplicada a técnica de conveniência para a coleta. Outra limitação se deu em virtude de que neste estudo não foram considerados os gatilhos que poderiam contribuir para o surgimento do comportamento suicida, restringindo a interpretação mais direcionada dos achados. Um outro aspecto se refere ao fato de não ter sido considerada a orientação sexual da amostra, o que pode ter interferido nas análises envolvendo o sexo e as escalas utilizadas. Sugere-se que novos estudos sejam realizados com base em ajustes ou mesmo correções de aspectos não controlados por esta pesquisa.

O interesse e a realização deste estudo buscaram reduzir a carência de material empírico sobre a ocorrência do comportamento suicida nessa população, bem como os fatores que possam contribuir para o seu surgimento. Os achados poderão servir como um sinal de alerta para que as instituições de ensino promovam ações preventivas e de enfrentamento para essas questões. Por fim, espera-se que os gestores das universidades se mobilizem para oferecer recursos que possam preparar os jovens universitários para as possíveis dificuldades que poderão enfrentar na academia, visto que os índices de óbito por suicídio nesta população têm crescido perigosamente.

\section{Referências}

Aftab, M. T., Naqvi, A. A., Al-Karasneh, A. F., \& Ghori, S. A. (2018). Impact of religiosity on subjective life satisfaction and perceived academic stress in undergraduate pharmacy students. Journal of Pharmacy \& Bioallied Sciences, 10 (4), 192. doi: 10.4103/JPBS.JPBS_65_18

ANDIFES - Associação Nacional dos Dirigentes das Instituições Federais de Ensino Superior, (2016). IV pesquisa do perfil socioeconômico e cultural dos estudantes de graduação. Disponível em http://www.andifes.org.br/iv-pesquisa-perfil-socioeconomico-e-cultural-dos-estudantes-de-graduacao/

Associação Brasileira de Psiquiatria. (2014). Suicídio: Informando para prevenir. Disponível em https://www.cvv.org.br/wpcontent/uploads/2017/05/ suicidio informado para prevenir abp 2014.pdf 
Becker, S. P., Holdaway, A. S., \& Luebbe, A. M. (2018). Suicidal behaviors in college students: Frequency, sex differences, and mental health correlates including sluggish cognitive tempo. Journal of Adolescent Health, 63 (2), 181 188. doi: 10.1016/j.jadohealth.2018.02.013

Bertolote, J. M., Mello-Santos, C. D., \& Botega, N. J. (2010). Detecção do risco de suicídio nos serviços de emergência psiquiátrica. Revista Brasileira de Psiquiatria, 32, 87-95. doi: 10.1590/S1516-44462010000600005

Braga, L. L., \& Dell'Aglio, D. D. (2013). Suicídio na adolescência: Fatores de risco, depressão e gênero. Contextos Clínicos, 6, 2-14. doi: 10.4013/ ctc. 2013.61 .01

CDC - Centers for Disease Control and Prevention. (2017). Preventing suicide: A technical package of policies, programs, and practices. Disponível em https:// www.cdc.gov/violenceprevention/pdf/suicideTechnicalPackage.pdf

Conselho Federal de Psicologia (2019). Quanto somos. Disponível em http:// www2.cfp.org.br/infografico/quantos-somos/

Conte, M., Meneghel, S. N., Trindade, A. G., Ceccon, R. F., Hesler, L. Z., Cruz, C. W., ... \& Jesus, I. (2012). Programa de prevenção ao suicídio: Estudo de caso em um município do sul do Brasil. Ciência \& Saúde Coletiva, 17, 2017-2026. doi: 10.1590/S1413-81232012000800013.

Dransart, D. (2018). Spiritual and religious issues in the after math of suicide. Religions, 9(5), 153-171. doi: 10.3390/rel9050153

Espinha, D. C. M., Camargo, S. M., Silva, S. P. Z., Pavelqueires, S., \& Lucchetti, G. (2013). Opinião dos estudantes de enfermagem sobre saúde, espiritualidade e religiosidade. Revista Gaúcha de Enfermagem, 34 (4), 98-106. doi: 10.1590/S1983-14472013000400013

Fleury, L. F. D. O., Gomes, A. M. T., Rocha, J. C. C. C. D., Formiga, N. S., Marques, S. C., \& Bernardes, M. M. R. (2018). Religiosidade, estratégias de coping e satisfação com a vida: Verificação de um modelo de influência em estudantes universitários. Revista Portuguesa de Enfermagem de Saúde Mental, 20, 51-57. doi: 10.19131/rpesm.0226

Jahn, G. M., \& Dell'Aglio, D. D. (2017). A religiosidade em adolescentes brasileiros. Revista de Psicologia da IMED, 9, 38-54. doi: 10.18256/21755027.2017.v9i1.1541

Jongkind, M., Van den Brink, B., Schaap $\square$ Jonker, H., Van der Velde, N., \& Braam, A. W. (2018). Dimensions of religion associated with suicide attempt and suicide ideation in depressed, religiously affiliated patients. Suicide and Life $\square$ Threatening Behavior, 49 (2), 505-519.doi: 10.1111/sltb.12456

Koenig, H. G. (2012). Religion, spirituality, and health: The research and clinical implications. ISRN Psychiatry, 2012, 1-33. doi: 10.5402/2012/278730

Koenig, H., Parkerson Júnior, G. R., \& Meador, K. G. (1997). Religion index for psychiatric research. The American Journal of Psychiatry, 154(6), 885-886. doi: 10.1176/ajp.154.6.885b

Kralovec, K., Fartacek, C., Fartacek, R., \& Plöderl, M. (2014). Religion and suicide risk in lesbian, gay and bisexual Austrians. Journal of Religion and Health, 53 (2), 413-423. doi: 10.1007/s10943-012-9645-2

Lamis, D. A., Malone, P. S., \& Jahn, D. R. (2014). Alcohol use and suicide proneness in college students: A proposed model. Mental Health and Substance Use, 7, 59-72. doi: 10.1080/17523281.2013.781535

Lawrence, R. E., Brent, D., Mann, J. J., Burke, A. K., Grunebaum, M. F., Galfalvy, H. C., \& Oquendo, M. A. (2016). Religion as a risk factor for suicide attempt and suicide ideation among depressed patients. The Journal of Nervous and Mental Disease, 204 (11), 845-850. doi: NMD.0000000000000484

Lawrence, R. E., Oquendo, M. A., \& Stanley, B. (2016). Religion and suicide risk: A systematic review. Archives of Suicide Research, 20, 1-21. doi: 10.1080/13811118.2015.1004494
Macedo, J. P., Alves, C. D. S., Bezerra, L. L. D. S., \& Silva, J. R. D. (2018). A "popularização" do perfil dos estudantes de psicologia no Brasil. Arquivos Brasileiros de Psicologia, 70 (2), 81-95. Disponível em http://pepsic.bvsalud. org/pdf/arbp/v70n2/07.pdf

McCabe, R., Garside, R., Backhouse, A., \& Xanthopoulou, P. (2016). Effective communication in eliciting and responding to suicidal thoughts: a systematic review protocol. Systematic Reviews, 5, 31-36. doi: 10.1186/s13643-016-0211-y

Ministério da Saúde (2019). Boletim epidemiológico Suicídio: tentativas e óbitos por intoxicação exógena no Brasil, 2007 a 2016. Disponível em https://www. saude.gov.br/images/pdf/2019/julho/17/2019-014-Publicacao-02-07.pdf

Oliveira, M. R., \& Junges, J. R. (2012). Saúde mental e espiritualidade/religiosidade: A visão de psicólogos. Estudos de Psicologia, 17 (3), 469-476. doi: 10.1590/S1413-294X2012000300016

O’Neill, S., McLafferty, M., Ennis, E., Lapsley, C., Bjourson, T., Armour, C., \& Murray, E. (2018). Socio-demographic, mental health and childhood adversity risk factors for self-harm and suicidal behaviour in College Students in Northern Ireland. Journal of Affective Disorders, 239, 58-65. doi: 10.1016/j.jad.2018.06.006

Osman, A., Bagge, C. L., Gutierrez, P. M., Konick, L. C., Kopper, B. A., \& Barrios, F. X. (2001). The suicidal behaviors questionnaire-revised (SBQ-R): Validation with clinical and nonclinical samples. Assessment, 8(4), 443-454. doi: $10.1177 / 107319110100800409$

Pereira, A., \& Cardoso, F. (2015). Ideação suicida na população universitária: Uma revisão da literatura. Revista E-Psi, 5(2), 16-34. Disponível em https:// revistaepsi.com/wpcontent/uploads/artigos/2015/Ano5-Volume2-Artigo2. pdf

Salas-Wright, C. P., Lombe, M., Nebbitt, V. E., Saltzman, L. Y., \& Tirmazi, T. (2018). Self-efficacy, religiosity, and crime: profiles of African American youth in urban housing communities. Victims \& Offenders, 13, 84-101. doi: 10.1080/15564886.2016.1268986

Santos, H. G. B., Marcon, S.R., Espinosa, M.M., Baptista, M.N., \& Paulo, P.M.C. (2017). Fatores associados à presença de ideação suicida entre universitários. Revista Latino-Americana de Enfermagem, 25, e2878. doi: $0.1590 / 1518-8345.1592 .2878$

Santos, W. S. D., Ulisses, S. M., Costa, T. M. D., Farias, M. G., \& Moura, D. P. F. D. (2016). A influência de fatores de risco e proteção frente à ideação suicida. Psicologia, Saúde \& Doenças, 17 (3), 515-526. doi: 10.15309/16psd170316

Taunay, T. C. D. E., Gondim, F. D. A. A., Macêdo, D. S., Moreira-Almeida, A., Gurgel, L. D. A., Andrade, L. M. S., \& Carvalho, A. F. (2012). Validity of the Brazilian version of the duke religious index (DUREL). Archives of Clinical Psychiatry, 39(4), 130-135. doi: 10.1590/S0101-60832012000400003.

Wasserman, D., Thanh, H. T. T., Minh, D. P. T., Goldstein, M., Nordenskiöld, A., \& Wasserman, C. (2008). Suicidal process, suicidal communication and psychosocial situation of young suicide attempters in a rural Vietnamese community. World Psychiatry, 7, 47-53. doi: j.2051-5545.2008.tb00152.x

World Health Organization. (2014). Preventing suicide: A global imperative. Disponível em https://www.who.int/mental health/suicide-prevention/ world report 2014/en/

You, Z., Chen, M., Yang, S., Zhou, Z., \& Qin, P. (2014). Childhood adversity, recent life stressors and suicidal behavior in Chinese college students. PloSOne, 9 (3), e86672. doi: 10.1371/jornal.pone.0101612

Submetido em: 24-3-2020

Aceito em: 20-9-2020 